\title{
miR-29a-3p/T-bet Regulatory Circuit Is Altered in T Cells of Patients With Hashimoto's Thyroiditis
}

\author{
Stana Tokić1,2*t, Mario Štefanić3t, Ljubica Glavaš-Obrovac ${ }^{1}$, Amit Kishore ${ }^{2}$, \\ Zdenka Navratilova ${ }^{2}$ and Martin Petrek ${ }^{2}$ \\ ${ }^{1}$ Department of Medical Chemistry, Biochemistry and Clinical Chemistry, Faculty of Medicine, University of Osijek, \\ Osijek, Croatia, ${ }^{2}$ Department of Pathological Physiology, Palacky University and Faculty Hospital, Olomouc, Czechia, \\ ${ }^{3}$ Department of Nuclear Medicine and Oncology, Faculty of Medicine, University of Osijek, Osijek, Croatia
}

Objective: Hashimoto's thyroiditis $(\mathrm{HT})$ is a common autoimmune thyroid disorder that frequently evolves from asymptomatic, T-cell mediated chronic inflammation toward overt hypothyroidism. Previously, we have demonstrated a role for T-bet, a $T$ helper $1 / \mathrm{CD}^{+} \mathrm{T}$ cell transcription factor (TF), and FoxP3, a regulatory $T$ cell TF, in disease progression and severity, but the basis behind their altered mRNA expression remains

OPEN ACCESS

Edited by: Alessandro Antonelli, Università degli Studi di

Pisa, Italy

Reviewed by:

Pieter de Lange,

Università degli Studi della

Campania "Luigi Vanvitelli"

Caserta, Italy

Flavia Di Bari,

Policlinico di Messina, Italy

*Correspondence:

Stana Tokić

stokic@mefos.hr

tThese authors have contributed equally to this work.

Specialty section:

This article was submitted to

Thyroid Endocrinology,

a section of the journal

Frontiers in Endocrinology

Received: 12 January 2018

Accepted: 07 May 2018

Published: 24 May 2018

Citation:

Tokić S, Štefanić M,

Glavaš-Obrovac L, Kishore A,

Navratilova Z and Petrek M (2018) miR-29a-3p/T-bet Regulatory

Circuit Is Altered in T Cells of

Patients With Hashimoto's

Thyroiditis.

Front. Endocrinol. 9:264. doi: 10.3389/fendo.2018.00264 unknown. In this study, we aimed to leverage the role for microRNAs, representing negative transcriptional regulators, across the spectrum of HT clinical presentations using the same, well-characterized RNA sample cohort.

Method: Ten hypothyroid, untreated patients (hypoHT), 10 hypothyroid cases rendered euthyroid by L-thyroxine therapy (substHT), 11 spontaneously euthyroid HT subjects (euHT), and 10 healthy controls (ctrl) were probed for three candidate immunoregulatory miRNA (miR-9-5p, miR-29a-3p, and miR-210-3p) using quantitative real-time PCR measurements. Data were normalized to U6snRNA and fold difference in expression calculated by the efficiency corrected $2^{-\Delta \Delta c t}$ model.

Results: Compared to healthy controls, peripheral blood (PB) T cells of HT patients exhibited significantly diminished miR-29a-3p expression levels [median expression levels (IQR), HT vs CTRL, 0.62 (0.44-1.01) vs 1.373 (0.63-2.7), $P=0.046]$, and a similar, but not significant decline in miR-210-3p abundance [HT vs CTRL, 0.64 (0.39-1.31) vs 1.2 (0.5-2.56), $P=0.24$, Wilcoxon test]. A significant inverse correlation was observed between the two differentially expressed transcripts, T-bet mRNA and miR-29a-3p. Moreover, altered miR-29a-3p/T-bet expression in T cells of untreated HT patients was related to low serum $\mathrm{FT} 4$, high serum thyrotropin, and decreased thyroid volumes. Of note, miR-210-3p expression was positively correlated to HIF1 $\alpha$, and inversely to FoxP3 mRNA levels, but no evidence of differential expression for any of these miRNA-mRNA pairs was observed. Finally, miR-9-5p expression levels were no different in HT vs control comparisons, or related to clinicopathological features.

Conclusion: T cell miR-29a-3p is downregulated in HT patients and associated with clinical and biochemical parameters of progressive thyroid injury, plausibly subsequent to altered control of T-bet expression in PB T cells. As such miR-29a-3p/T-bet axis should be further explored as a biomarker or as a plausible target for therapeutic interventions in HT.

Keywords: Hashimoto disease, T-lymphocytes, hsa-miR-29a, hsa-miR-210, hsa-miR-9, disease attributes, thyroid gland 


\section{INTRODUCTION}

Hashimoto's thyroiditis (HT) is a multifactorial, autoimmune disorder characterized by the presence of thyroid-specific autoantibodies, chronic lymphocytic infiltration of the thyroid gland, and, eventually, hypothyroidism (1). Pathologically, HT is mediated by aberrant T helper type 1 (Th1), Th17, Treg, and cytotoxic (Tc) $\mathrm{CD}^{+}$responses, which typically require master regulator transcription factors (TFs) T-bet, ROR $\gamma$ t, FOXP3, and EOMES, respectively, for their development. Of these, T-bet $(2,3)$, ROR $\gamma \mathrm{t}(4-6)$, and FOXP3 $(3,7,8)$ have all been implicated in the ontogeny and severity of HT in humans; however, the mechanisms beyond the reported deregulated expression of these TFs in patients' $\mathrm{T}$ cells remain unclear.

Certain microRNAs (miRNAs, miR) act by negatively regulating the expression of key master regulators within this network (9). miRNAs are a class of single-stranded 19-25 nucleotide long non-coding RNAs that bind 3 '-untranslated region of target mRNA and result in either translation inhibition or mRNA degradation. Among these, miR-29a-3p limits Th1/ Tc1 bias by targeting interferon (IFN)- $\gamma$-inducing TFs EOMES and T-bet (10). In Treg cells, miR-9 and miR-210 are markedly downregulated in $\mathrm{CD} 4^{+} \mathrm{CD} 25^{+} \mathrm{CD} 127^{\text {low }}(11)$ and $\mathrm{CD} 8^{+} \mathrm{CD} 25^{+}$ (12) subsets compared to non-Treg T cells. By contrast, miR210 , the hypoxia-induced $\mathrm{miR}$, is greatly enhanced in activated, effector $\mathrm{CD}^{+}$and $\mathrm{CD} 4^{+} \mathrm{T}$ cells under Treg- $>\mathrm{Th} 17$-polarizing conditions (13), whereby it inhibits FOXP3 expression and impairs the immunosuppressive functions of $\mathrm{CD} 4^{+}$Treg cells. HIF1 $\alpha$, a TF-controlling Th17 polarization and $\mathrm{CD}^{+}$effector differentiation (14), is both an upstream miR-210 regulator and a miR-210 target, thus; miR-29, miR-210, and miR-9, all seem to affect a broad spectrum of differentiation pathways, including those responsible for producing effector and regulatory cells. Nevertheless, despite evidence supporting the importance of these miRs in controlling disease-associated TFs, their respective regulatory networks in the context of HT, disease severity, and clinical presentation remain mostly unexplored.

We previously described increments in transcript levels of Th1 and Treg-associated, T-bet, and FOXP3, but not Th17-related HIF $1 \alpha$ TF, in peripheral blood (PB) T cells of severely affected HT patients. In this study, the same RNA sample cohort was probed for expression levels of their respective miRNA pairs. Thus, we assessed the transcriptional patterns of miR-29-3p, miR-210-3p, and miR-9-5p in bulk PB T cells and attempted to combine the selected TFs and miRNAs to study their co-regulatory networks, and effects on clinical features of HT patients with distinct patterns of disease severity.

\section{MATERIALS AND METHODS}

\section{Subjects}

In this study, we built upon our previous work by screening for selected miRNAs in an established and well-characterized cohort of HT cases and healthy controls who had been previously typed for T-bet, FoxP3, BLIMP1, and HIF1 $\alpha$ expression in bulk PB T cells. HT was classified as (1) hypothyroid, untreated [hypoHT, $n=10,2$ males], (2) spontaneously euthyroid HT
[euHT, $n=12$, all females], and (3) rendered euthyroid by hormone replacement therapy [substHT, $n=10,1$ male, median L-thyroxine (T4) dose $1.13 \mu \mathrm{g} / \mathrm{kg}$ body mass daily, median pretreatment serum thyrotropin (TSH) $15.1 \mathrm{mU} / \mathrm{L}$, interquartile range $11.5-35.3 \mathrm{mU} / \mathrm{L}]$. HT was defined and exclusion criteria applied as previously described (15). Healthy, euthyroid control subjects $(n=10,1$ male), had normal ultrasound findings of the thyroid gland and were negative for thyroid autoantibodies. All participants were unrelated adults from eastern Croatia. This study protocol was reviewed and approved by the institutional ethical committee of the Osijek University Hospital, and all subjects gave written informed consent prior to the testing.

\section{Thyroid Function Measurement}

TSH (normal range: 0.46-4.7 mIU/L, Vitros TSH Reagent Pack), free tri-iodothyronine (FT3) (1.9-5.7 pmol/L, Vitros FT3 Reagent Pack), and free T4 (FT4, 10-22 pmol/L, Vitros FT4 Reagent Pack, all from Ortho-Clinical Diagnostics, Amersham, UK) were measured in sera taken between 8 and 12 a.m., according to the manufacturer's instructions. Maximum pretreatment TPOAb$\operatorname{IgG~(50-125~kIU/L)~was~determined~by~ELISA~kit~(Anti-TPO,~}$ MileniaBiotec, Germany) calibrated against WHO reference MRC 66/387a. Hypothyroidism was defined as clinically significant when TSH was $>4.7 \mathrm{mU} / \mathrm{L}$ and FT4 was $<10 \mathrm{pmol} / \mathrm{L}$, but it was considered subclinical or latent if TSH was $>4.7 \mathrm{mU} / \mathrm{L}$ and FT4 was $>10 \mathrm{pmol} / \mathrm{L}$.

\section{Thyroid Volume Measurements}

The thyroid volume was sonographically established as the sum of the volumes of the two lobes using a $10 \mathrm{MHz}$ linear array transducer (Accuson X-150, Siemens, Germany). Each lobe was assumed an ovoid $(\pi / 6 \times$ length $\times$ width $\times$ depth $)$ as previously specified (16).

\section{Peripheral Blood Mononuclear Cells (PBMC) Isolation}

Peripheral blood mononuclear cells were isolated from freshly collected heparinized blood samples using density gradient centrifugation on LymphoPrep (Axis Shield, Oslo, Norway) as described in details elsewhere (16). Briefly, $10 \mathrm{~mL}$ initial volume of whole blood was diluted with $0.9 \%(\mathrm{w} / \mathrm{v}) \mathrm{NaCl}$ in 1:1 ratio, carefully layered over $20 \mathrm{~mL}$ LymphoPrep medium and sedimented into fractions during $20 \mathrm{~min}$ centrifugation at $800 \times g$. Fraction of mononuclear cells retained at the plasma/medium interface was harvested, rinsed with saline, and spun down for $10 \mathrm{~min}$ at $550 \times g$. Pooled cells were further rinsed in two successive wash cycles. Final PBMC pellet was resuspended with $1 \mathrm{~mL}$ of isolation buffer (PBS without $\mathrm{Ca}^{2+} \mathrm{i} \mathrm{Mg}^{2+}$ with $0.1 \%$ (v/v) BSA and $2 \times 10^{-3} \mathrm{~mol} / \mathrm{dm}^{3} \mathrm{EDTA}$ ) and cells were counted after trypanblue staining using the Bürker-Türk counting chambers and light microscope. A minimum of $1 \times 10^{7}$ purified PBMCs were used in the following isolation step.

\section{Lymphocyte Subsets Separation}

Peripheral T-lymphocytes were separated from PBMCs by immunomagnetic depletion of non- $\mathrm{CD}^{+}$cells in cell suspension using DynaMag magnet and Dynabeads Untouched 
Human $\mathrm{T}$ cell Isolation Kit (Invitrogen, Paisley, UK), as described previously (3). In the first $20 \mathrm{~min}$ incubation step, $1 \times 10^{7} \mathrm{PBMCs}$ resuspended in $100 \mu \mathrm{L}$ isolation buffer enriched with $20 \%(\mathrm{v} / \mathrm{v})$ FBS, were labeled with mouse monoclonal antibodies specific for CD14, CD16, CD19, CD36, CD56, CDw123, and CD235 markers. Antibody-labeled cells and unstained $\mathrm{CD}^{+}$T-lymphocytes were rinsed, carefully mixed by tilting, and pooled down by centrifugation for $8 \mathrm{~min}$ at $300 \mathrm{~g}$. Pelleted cells were resuspended in $100 \mu \mathrm{L}$ of isolation buffer and incubated for $15 \mathrm{~min}$ with pre-washed magnetic beads, coated with human anti-mouse IgG antibody. Bead-bound cells adhered to the polypropylene tube walls when exposed to the DynaMag stationary magnetic field, leaving cell suspension free from B-lymphocytes, natural killer cells, monocytes, platelets, dendritic cells, granulocytes, and erythrocytes. Non-adherent $\mathrm{CD}^{+}$and $\mathrm{CD}^{+} \mathrm{T}$ cells were gently removed by pipetting to a new tube. Cell separation procedures were repeated in two successive cycles of immunomagnetic selection and washing. The final $T$ cell collection was purified from the residual Dynabeads by placing the tube in a magnet for $2 \mathrm{~min}$ and then transferring the supernatant to a fresh tube.

\section{Total RNA Extraction}

Extraction of total RNA was performed with the use of TRI REAGENT (Sigma, USA) following the single-step technique as described by Chomczinsky and Sacchi (17). The RNA integrity was examined by ethidium bromide staining in $2 \%$ agarose gel electrophoresis. Quantity and purity of RNA samples was checked by NanoDrop 1000 spectrophotometer (Thermo Fisher Scientific, USA) and verified by $\mathrm{OD}_{260} / \mathrm{OD}_{280}$ ratio $>1.8$ measurements.

\section{Reverse Transcription and microRNA Real-Time PCR Quantification}

Sequence-specific stem loop reverse transcriptase (RT) primers and TaqMan MicroRNA Reverse Transcription Kit were used for cDNA synthesis of three candidate miRNA (miR-9-5p, miR$29 a-3 p$, and miR-210-3p) and two reference small nuclear RNA
(U6snRNA and RNU48) according to the TaqMan MicroRNA assay protocol (PE Applied Biosystems, Foster City, CA, USA). Reverse reactions were performed with $50 \mathrm{ng}$ of total RNA in a $15 \mu \mathrm{L}$ final volume comprising $1 \times \mathrm{RT}$ buffer, $1 \mathrm{mM} \mathrm{dNTP}$ each, $3.33 \mathrm{U} / \mu \mathrm{L}$ MultiScribe RT, $0.25 \mathrm{U} / \mu \mathrm{L}$ RNase inhibitor, and $3 \mu \mathrm{L}$ of $5 \times$ RT primer. Reaction mixture was incubated for $30 \mathrm{~min}$ at $16^{\circ} \mathrm{C}, 30 \mathrm{~min}$ at $42^{\circ} \mathrm{C}$, and $5 \mathrm{~min}$ at $85^{\circ} \mathrm{C}$, next diluted eightfold and stored in aliquotes at $-20^{\circ} \mathrm{C}$ until use.

miRNA transcript levels were measured using the Rotor Gene 3000 instrument (Corbett Research, USA) in triplicate $15 \mu \mathrm{L}$ quantitative real-time PCR (qRT-PCR) reactions containing $5.0 \mu \mathrm{L}$ of cDNA, $7.5 \mu \mathrm{L}$ of TaqMan Universal PCR Master Mix II kit, and $0.75 \mu \mathrm{L}$ of pre-developed TaqMan miRNA expression assay (Applied Biosystems). The cycling conditions were set according to the guidelines in the manufacturer's leaflet and the list of assays is given in Table $\mathbf{1}$.

Ct values were collected at the fractional cycle number at which fluorescence passes fixed threshold of 0.05 . The linear regression coefficient $\left(R^{2}\right)$ and amplification efficiency were assessed through five-point fourfold serial dilutions of the arbitrary standards prepared individually for each miRNA assay, and final values ranged between $0.994-0.998$ and $92-100 \%$, respectively. Intra-assay variability was less than $1.12 \%$, and less than $2.01 \%$ variation was achieved between different PCR experiments. Prior to qPCR data normalization, two small nuclear RNA were tested for stability using geNorm and NormFinder algorithms. Both U6snRNA and RNU48 endogenous controls expressed the same measure of stability $(M=1.046)$, but U6snRNA was used for miRNA gene normalization due to the lower inter-group variability $(\mathrm{SD} \pm 0.15)$ when compared to RNU48 (SD \pm 0.22 ). Fold difference in miRNA expression was calculated using efficiency corrected model of $2^{-\Delta \Delta \mathrm{Ct}}$ method as described by Pfaffl (18). Data are presented as relative quantity of target miRNA, normalized with respect to U6snRNA and a control group (19). A detailed description of T-bet, FoxP3, BLIMP1, and HIF1 $\alpha$ mRNA quantification analysis, together with PCR efficiency results and stability measurements of validated housekeeping genes, can be found elsewhere (3).

TABLE 1 | List of TaqMan assays of investigated miRNA.

\begin{tabular}{|c|c|c|c|c|c|}
\hline Assay ID & miRBase ID & $\begin{array}{l}\text { miRBase accession } \\
\text { number }\end{array}$ & Mature miRNA sequence & Function & Target \\
\hline 000583 & hsa-miR-9-5p & MIMAT0000441 & UCUUUGGUUAUCUAGCUGUAUGA & $\begin{array}{l}\text { Downregulate NF-KB, enhances } \\
\text { IL-2 production in activated } \\
\text { human CD4(+) T cells }\end{array}$ & BLIMP1 \\
\hline 002112 & hsa-miR-29a-3p & MIMAT0000086 & UAGCACCAUCUGAAAUCGGUUA & $\begin{array}{l}\text { Inhibitor of Th1 development } \\
\text { and interferon (INF)- } \gamma \text { expression }\end{array}$ & INF- $\gamma$, T-bet, EOMES \\
\hline 001006 & $\mathrm{RNU} 48$ & NR_002745 & $\begin{array}{l}\text { AGTGATGATGACCCCAGGTAACTC } \\
\text { TGAGTGTGTCGCTGATGCCATCAC } \\
\text { CGCAGCGCTCTGACC }\end{array}$ & Control miRNA assay & \\
\hline 001973 & U6 snRNA & NR_004394 & $\begin{array}{l}\text { GTGCTCGCTTCGGCAGCACATATACT } \\
\text { AAAATTGGAACGATACAGAGAAGATTA } \\
\text { GCATGGCCCCTGCGCAAGGATGACAC } \\
\text { GCAAATTCGTGAAGCGTTCCATATIT }\end{array}$ & Control miRNA assay & \\
\hline
\end{tabular}




\section{Statistical Analysis}

Normality of distributions was tested by Shapiro-Wilk test. Given that data used in this study were often not normally distributed, nonparametric approaches were selected, in general. Proportions and median with interquartile range (IQR) were used for presentation of data. Exact binomial test, Wilcoxon test, and Kruskal-Wallis test with Bonferroni-corrected Dunn's post hoc analysis were used for group comparisons. Correlations between paired datasets were determined by Spearman rank-test. Twotailed $P<0.05$ was considered significant. If not otherwise stated, statistical analyses were performed with NCSS2007 program (v07.1.20, NCSS LLC, Kaysville, UT, USA).

\section{RESULTS}

\section{HT Patients Characteristics: Biochemical and Clinical Data}

Patients and healthy controls were matched for sex and age (Table 2). No difference in FT3 or TSH serum levels was observed between healthy controls, euthyroid, and L-T4 treated HT subjects; however, LT4-receiving patients required higher serum FT4 levels to attain comparable serum FT3 levels. Untreated, hypothyroid subjects demonstrated the opposite trend showing lower FT4, but higher serum TSH levels in comparison to control and LT4 substituted group. FT3 levels increased with the serum FT4 ( $\rho=0.313, P=0.043)$ concentrations in pooled sample of HT patients and controls, and declined with age in HT cases $(\rho=-0.358, P=0.045)$.

\section{PB T Cells From HT Patients Display Reduced Expression of miR-29a}

We analyzed the expression levels of miR-29a, miR-210, and miR-9 using RNA bank from the prior cohort as well as using published qRT-PCR data on selected target mRNAs from our previous study (3). Because no difference in expression levels of candidate miRNAs was present across different HT stages, we further analyzed HT data as one group.

Subsequently, miR-29a-3p expression levels were significantly diminished in bulk PB T cells of HT patients \{median fold difference 0.62 [IQR (0.44-1.01), $P=0.046$, Wilcoxon test, Figure 1A, $n=32]\}$ when compared to their healthy counterparts [1.373 (0.63-2.7), $n=10]$. A similar, but not significant trend was observed for miR-210-3p in HT vs control comparison [HT vs ctrl; $0.64(0.39-1.31)$ vs $1.2(0.5-2.56), P=0.24$, Figure 1B]. Finally, no change in expression levels of miR-9-5p was seen between HT cases [0.64 (0.49-1.02) vs 1.08 (0.54-1.92), $P=0.26$, Figure 1C] and healthy subjects.

\section{miRNA-mRNA Target Pairs in PB T Cells From HT Patients}

To gain further insight into the miRNA-target RNA dynamics, we integrated data from previously collected mRNA measurements with the newly obtained miRNA findings. A number of significant correlations were observed in pooled HT and control samples, majority of which was consistent with the previously reported post-transcriptional mechanisms of miR-29 (10) and miR-210 (11) action. Importantly, T-bet (Spearman's correlation coefficient, $\rho=-0.389, P=0.011, n=42$, Figure 2A) mRNA levels increased with declining miR-29a-3p levels, whereas FOXP3 transcript abundance increased with diminished miR-210-3p levels $(\rho=-0.409, P=0.0071, n=42$, Figure 2B). The associations persisted upon exclusion of control samples (data not shown); thus eliminating the confounding by case-control status. In addition, miR-210-3p seemed to be associated with HIF1 $\alpha$ regulatory circuits, being positively related to HIF1 $\alpha$ transcript abundance in the HT group ( $\rho=0.397, P=0.025, n=32$, Figure 2C).

\section{T-bet, miR-29a-3p, and miR-210-3p Transcript Levels Are Associated With the Clinical Features of HT}

In HT patients, residual thyroid volume was negatively associated with increase in T-bet $(\rho=-0.417, P=0.02, n=32$, Figure 3A) and BLIMP1 mRNA transcript levels $(\rho=-0.389$, $P=0.03, n=32$, Figure 3B) of PB T cells, particularly in euthyroid $($ euHT + substHT $)$ cases $[\rho(T-b e t)=-0.483, P=0.027$; $\rho(B L I M P 1)=-0.558, P=0.0085]$. Moreover, increased T-bet mRNA expression was associated with reduced serum FT4 levels ( $\rho=-0.45, P=0.036, n=22$, Figure 3C) and increased TSH levels $(\rho=0.554, P=0.0075$, Figure 3D) among the therapeutically

TABLE 2 | Descriptive analysis of clinical and biochemical characteristics of patients and healthy controls.

\begin{tabular}{|c|c|c|c|c|c|}
\hline & HYPO Hashimoto's thyroiditis (HT) & SUBST HT & EU HT & CTRL & $P^{*}$ \\
\hline Subjects $(n)$ & 10 & 10 & 12 & 10 & - \\
\hline Age (years) & $45(26-56)$ & $64(61-65)$ & $51(41-60)$ & $44(36-59)$ & 0.069 \\
\hline Gender (F/M) & $8 / 2$ & $9 / 1$ & $12 / 0$ & $9 / 1$ & - \\
\hline FT4 (pmol/L) & $11.4(10.2-12.5)^{\star \star}$ & $15.4(12.2-17.6)^{\#}$ & $11.8(11.3-13)$ & $13.8(12.4-14.6)$ & 0.000054 \\
\hline FT3 (pmol/L) & $2.92(2.46-3.14)$ & $2.83(2.43-3.55)$ & $2.9(2.45-4.04)$ & $3.38(2.75-3.87)$ & 0.173 \\
\hline TSH (mlU/L) & $9.6(5.59-13.1)$ & $2.6(1.19-3.25)^{\# \#}$ & $3.11(1.65-4.04)^{\# \#}$ & $1.65(0.98-2.62)^{\# \#}$ & $<0.000001$ \\
\hline Volume (mL) & $16(14.1-19.4)$ & $11.1(6.9-13.9)$ & $14.6(11.2-20.5)$ & $11.9(10.2-12.8)$ & 0.136 \\
\hline TPOAb (kIU/mL) & $155(61-3,000)$ & $260(150-1,355)$ & $690(272-2,528)$ & Neg & - \\
\hline
\end{tabular}

*Kruskal-Wallis test.

${ }^{* *} P<0.05$ vs CTRL.

"P $<0.05$ vs HYPO HT and EU HT.

$\# P<0.05$ vs HYPO HT.

FT4, free thyroxine; FT3, free tri-iodothyronine; TSH, thyroid stimulating hormone; TPOAb, thyroid peroxidase antibodies. 


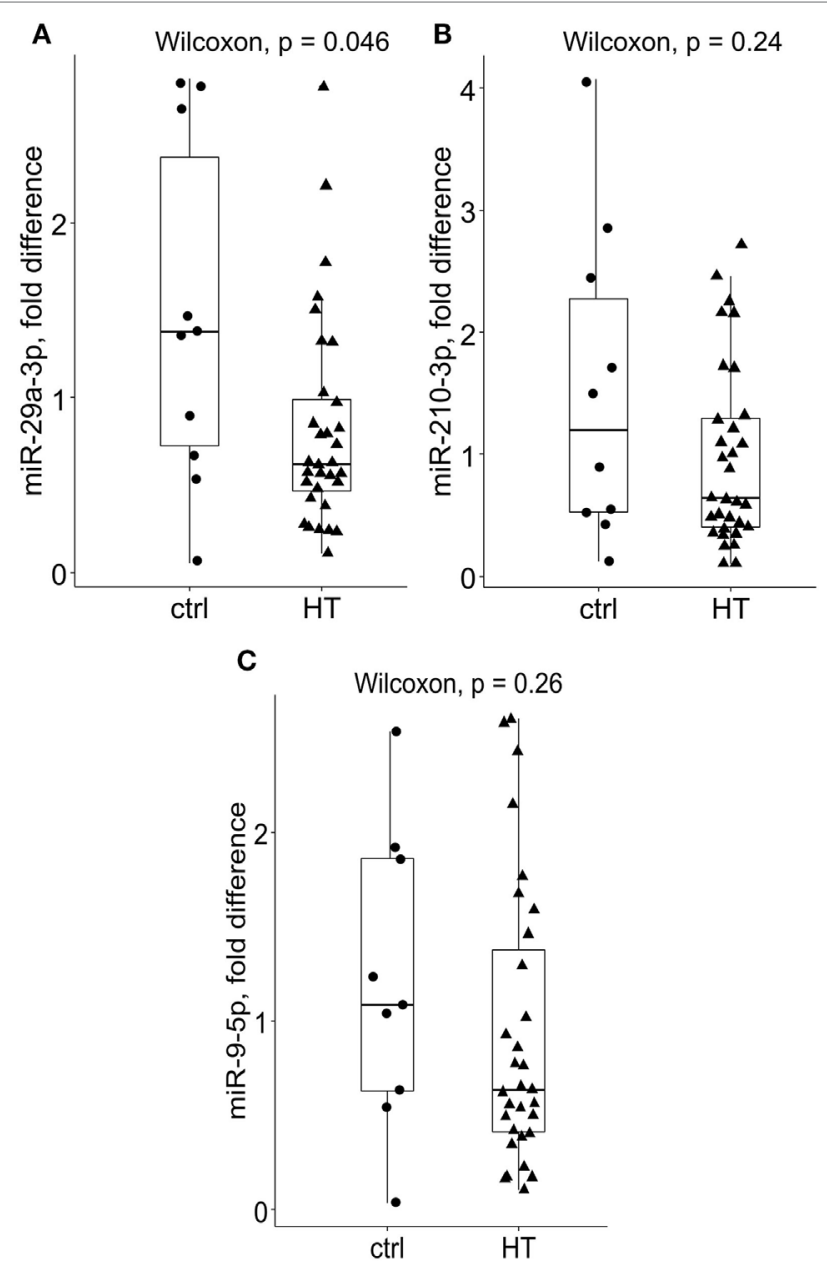

FIGURE 1 | Relative expression levels of miR-29a-3p, miR-210-3p, and miR-9-5p in Hashimoto's thyroiditis $(H T)$ patients and healthy controls. Compared to controls, (A) miR-29a-3p levels were significantly reduced in bulk peripheral blood T cells of HT patients [HT $(n=32), P=0.046$, Wilcoxon test, Bonferroni-Dunn's post hoc comparison]. A non-significant decrease in (B) miR-210-3p levels was noted in HT vs control comparisons (HT vs controls; 0.64 vs 1.2, $P=0.24)$. Conversely, no difference in (C) miR-9-5p expression levels was found across the studied groups. Transcriptional changes were measured by RT-qPCR and normalized against U6snRNA. Within each box, the horizontal line represents the median value, and the first and third quartiles are the ends of the box. The whiskers extend to $1.5 x$ (interquartile range).

naive patients, both spontaneously euthyroid and hypothyroid ones, independently of the individual thyroid volume [partial $\rho($ FT4 $)=-0.518, P=0.016$ ] and age [partial $\rho($ FT4 $)=-0.435$, $P=0.049]$. Conversely, miR-29a transcript levels were positively related to serum FT4 values $(\rho=0.473, P=0.026)$ in untreated HT cases $(n=22$, Figure 3E), whereas miR-210 levels were positively associated with FT3 levels $(\rho=0.44, P=0.041)$. Age and thyroid volume did not alter the relation.

By univariate analysis, serum FT3 levels were associated with miR-29a-3p $(\rho=0.39, P=0.0099)$ expression and T-bet ( $\rho=-0.439, P=0.0037)$ transcript abundance in pooled dataset of HT cases and controls $(n=42)$. The associations persisted upon correction for FT4 levels [partial $\rho(\operatorname{miR}-29 a-3 p)=0.337$, $P=0.031, \rho(T-b e t)=-0.39, P=0.012]$ and age [partial $\rho(\mathrm{miR}$ $29 \mathrm{a}-3 \mathrm{p})=0.428, P=0.0052, \rho(T-b e t)=-0.374, P=0.016]$. No relationship was seen between miR-9 and any of the tested clinical features. Furthermore, TPOAb levels and LT4 replacement dose were not related to any miRNA or mRNA analyzed.

\section{DISCUSSION}

We previously observed high T-bet and FoxP3 mRNA expression in PB T cells from HT patients relative to age-matched healthy control individuals. In this study, we looked for evidence of correlated changes in miRNA-target mRNA profile by measuring transcript levels of miR-9-5p, miR-29a-3p, and miR-210-3p in an identical RNA sample set. We could now demonstrate that miR-29a-3p may be involved in the imbalance of T-bet through decreased miR-29a-3p expression in T cells of HT patients, which may play an important role in the development of HT. In parallel, negative miR-210-3p/FoxP3 and positive miR-210-3p/HIF1 $\alpha$ circuits were also noted, possibly reflecting postranscriptional dynamics previously described in activated $\mathrm{CD} 4^{+}$and $\mathrm{CD} 8^{+}$effectors under conditions of low oxygen tension (14). Nevertheless, underlying miR-210-3p mechanisms in HT autoimmunity remain unclear, since we found no difference in miR-210-3p expression between HT and control subjects; a result supporting redundant or possibly diluted effects of miR-210-3p in mixed PB cell populations of HT patients. In contrast, downregulated miR-29-3p levels suggest a dysregulation of the miR-29/T-bet feedback loop, which may bias Th1 and Tc1 cell differentiation and contribute to chronic inflammation in HT.

The functional relevance of this altered T-bet/miR-29a-3p axis is further highlighted by finding that our untreated HT cohort with high T-bet/low miR-29a-3p expression had showed increased predisposition to develop thyroid insufficiency and primary hypothyroidism. Mechanistically, T4 and T3 synthesis are inhibited by cytokines directly on the level of thyroid epithelial cells (20): TSH-R gene is downregulated, and the expression of deiodinases (D) 1 and D2, which convert T4 into the most active metabolic form, T3, is altered (21). INF $\gamma$, a prototypical T-bet/miR-29 target, is the cytokine most clearly associated with thyroid injury, especially hypothyroidism $(22,23)$. Increased serum interleukin (IL)-2, TNF $\alpha$, and IFN $\gamma$ have been reported in subjects with overt hyopothyroidism due to HT $(24,25)$. Hashimoto's thyroids are enriched in intraglandular $\mathrm{CD} 4^{+} \mathrm{IFN} \gamma^{+}$and $\mathrm{CD} 8^{+} \mathrm{IFN} \gamma^{+} \mathrm{T}$ cells (26) and TPOAbs enhance the autoantigen-elicited production of IFN $\gamma$ by MNCs in a dose- and Fcy-receptor dependent manner (27). In addition, IL-12, which controls IFN $\gamma$ and T-bet expression in activated $\mathrm{T}$ cells, is another miR-29a target (28) that has been linked to hypothyroidism in AITD (29). Alternatively, or in addition; TSH, T4, and T3, in their own turn, can play an important role in $\mathrm{T}$ cells and thymus and regulate their functions via TSH, D2, and $\mathrm{T} 3$ receptors. No difference, however, in T-bet/miR-29a expression was observed between LT4-treated and untreated hypothyroid HT in this study, suggesting that the correction of FT4 by hormone replacement therapy alone does not prevent 

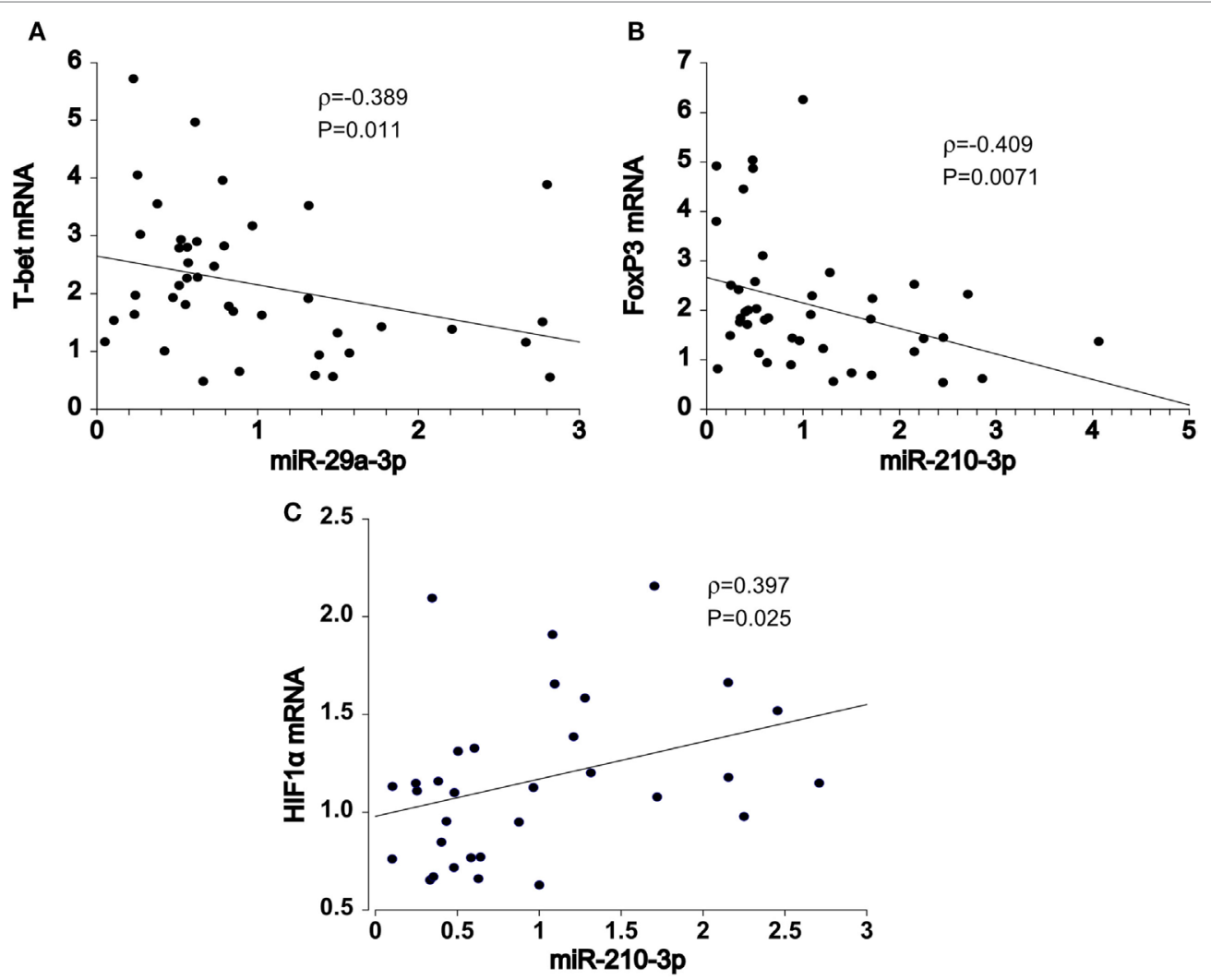

FIGURE 2 | Spearman pair-wise correlation analysis of miRNA-mRNA target pairs in pooled samples $(N=42)$. Declining (A) miR-29a-3p expression levels was associated with increments in T-bet transcript abundance. Similar, negative relationship was also noted between (B) miR-210-3p and FOXP3 mRNA levels. Conversely, (C) miR-210-3p was positively correlated with HIF1 $\alpha$ gene expression. Spearman rank-test, solid line: the least square estimate. $\rho-$ Spearman correlation coefficient; global significance $P<0.05$.

T-bet/miR-29a alterations in T cells from end-stage HT. Taken together, our data are in comply with studies showing no effect of low thyroid hormone levels on $\mathrm{T}$ cell cytokine production (30), but instead support a role for deregulated T-bet/miR-29a immunologic background in HT state.

The important role of T-bet in controlling HT symptoms in target organs is further complemented by observation linking transcriptional heterogeneity of T-bet and BLIMP1 gene in HT $\mathrm{PB} T$ cells with inter-individual variations in thyroid volume, which may also contribute to the progression of thyroid insufficiency and the development of hypothyroidism. Mechanistically, both BLIMP1 and T-bet are crucial factors for the development of short-lived effector cells (SLEC) and cytotoxic T lymphocyte (CTL) (31). In this context, a co-associated TF, BLIMP1, has been considered a major TF for the generation and function of cytotoxic CD4/CD8 and effector T cells. BLIMP1 acts by promoting the binding of T-bet to the promoters of cytolytic genes in $\mathrm{CD}^{+} \mathrm{T}$ cells and is required for the cytolytic function and effector differentiation of $\mathrm{CD} 4^{+} \mathrm{T}$ cells and $\mathrm{CD} 8^{+} \mathrm{CTLs}$, its expression peaking in antigen-experienced $\mathrm{T}$ cells (32). In vivo, inhibition of BLIMP1 reduces the production of autoantibodies and alleviates organ damage in selected autoimmune pathologies (33). By contrast, BLIMP1-deficient effector CTLs have impaired cytotoxicity, owing to their impaired expression of multiple cytotoxic molecules, including granzyme $\mathrm{B}$ and IFN $\gamma$, as well as the TF T-bet (34). Currently, however, the experimental link with thyroid injury yet remains to be established. Adding to the complexity of T-bet regulation, the transcriptional permissibility of Tbx 21 promoter has also been described in non-Th1/Tc1 subsets, including Th17 as well as iTreg cells. Several reports thus indicate that T-bet expression is essential for generation of Th17 cells and their switch from Th17 to highly pathogenic Th1-like exTh17 cells as well $(35,36)$. The tight regulation of T-bet is, therefore paramount, but little understood aspect of HT.

Regarding the potential source, miRNA expression levels dynamically change between naïve, effector, and memory $\mathrm{T}$ cell subsets (37). A global downregulation of miRNA, including miR-29a, has been seen in effector T cells, compared to naïve $\mathrm{T}$ precursors, and tends to increase back in memory $\mathrm{T}$ cells (38). SLEC are particularly sensitive to graded expression of T-bet and BLIMP1, and the formation of terminal effector cells is largely dependent on the IL-12/T-bet axis, which drives their generation in a dose-dependent manner (31). Thus, a more precise analysis of $\mathrm{T}$ cell subpopulations would offer a better definition of the miR-29a activity in HT T cells.

Hashimoto's thyroiditis is also characterized by altered composition of both FoxP3 ${ }^{+}$and Th17 cell compartments. HIF1 $\alpha$ 

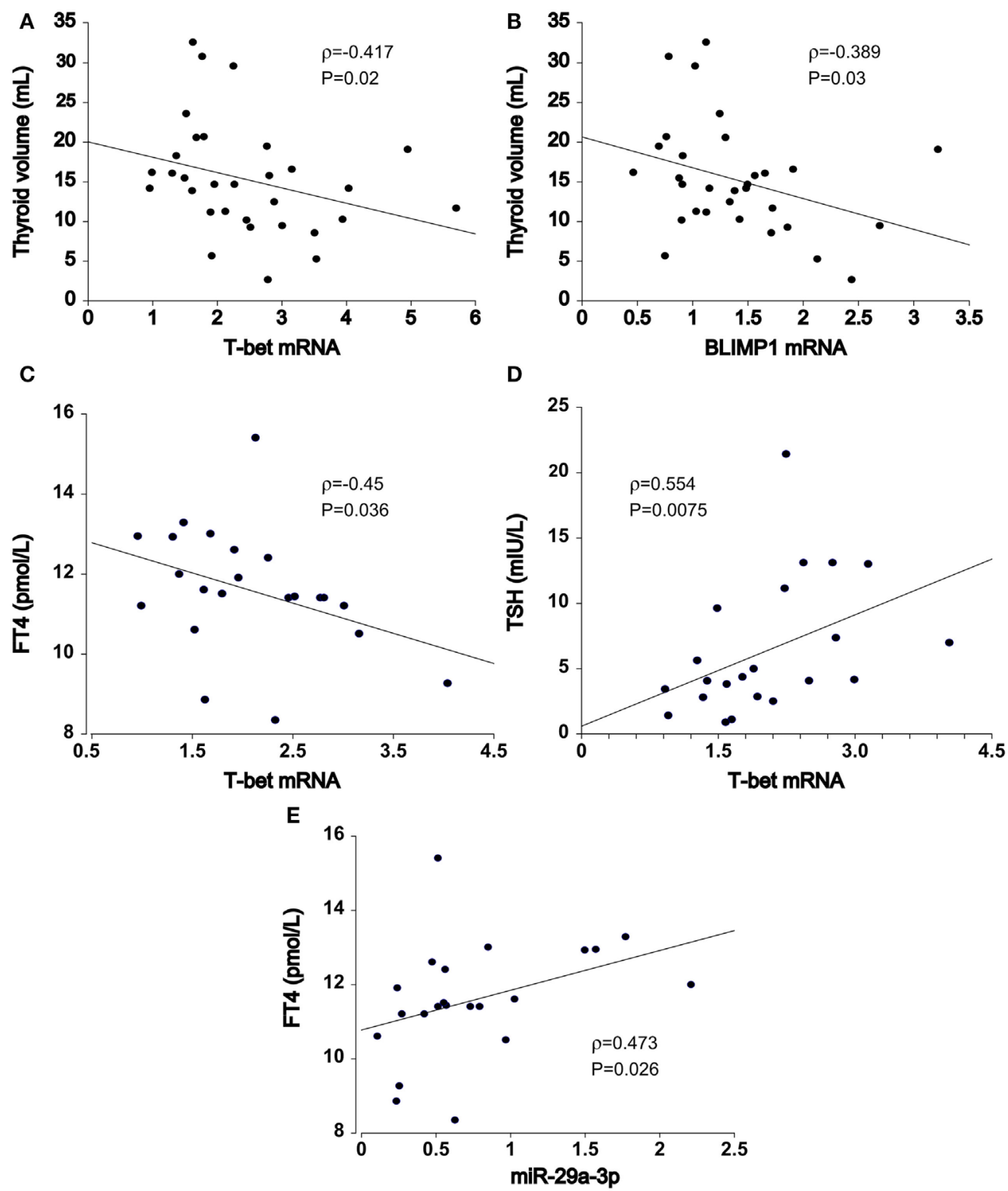

FIGURE 3 | Association of T cell miRNA and mRNA transcript levels with Hashimoto's thyroiditis (HT) clinical features. In HT patients ( $n=32$ ), thyroid volume was inversely related to increments in (A) T-bet and (B) BLIMP1 transcript abundance in peripheral blood T cells. In untreated HT patients (euthyroid + hypothyroid, $n=22$ ), upregulated (C) T-bet expression was associated with declining FT4 and (D) increasing TSH serum levels. (E) miR-29-3p expression exhibited opposite, positive effects in relation to serum FT4 levels. Transcriptional changes of mRNA and miRNA were measured by quantitative real-time PCR and normalized against TBP and U6snRNA, respectively.

was reported to control the balance of Th17/Treg differentiation through direct transcriptional activation of ROR $\gamma \mathrm{t}$; in addition, HIF1 $\alpha$ targets FOXP3 for proteasomal degradation, thereby inhibiting Treg development (14). HIF1 $\alpha$ acts partially by increasing the expression of miR-210 as well, which subsequently functions to inhibit both FoxP3 and HIF $1 \alpha$ in a negative feedback loop $(13,14)$. Consistent with these results, we observed a modest, but significant miR-target pairing in coexpression correlations, coupled to a non-significant decrease of miR-210 expression in PB T cells from HT patients. These moderate effects might nevertheless be sufficient to contribute to the regulatory alterations behind HT, as exemplified by fine-tuning quantitative effects typically exhibited by miRNAs (39). Positive or negative correlations alone, of course, do not prove causality, since there can be a plethora of different factors involved in mRNA degradation. One must also take into account that the comparison was made using an entire PB T cell population, so it is possible that dilution and confounding by mixed cell populations, notwithstanding a wide array of post-transcriptional mechanisms, may have acted to weaken the relation specific to a high-expressing fraction of $\mathrm{PB}$ cell population. Finally, it is possible that the repressive effect of selected miRs is more pronounced on the translational level. 
The mechanisms beyond miR-210 expression in HT thus remain unknown, and appear to play a limited role, at least under the current experimental settings. Similarly, the homogeneity of T cell populations, as well as the factors affecting the Treg purity and definition, is expected to affects the hierarchical expression of FOXP3 in HT, and therefore, the estimates of the miR-210 effect. Other, non-canonical $\mathrm{FOXP}^{+} \mathrm{T}$ cells, such as $\mathrm{FOXP} 3{ }^{+} \mathrm{CD} 127^{\text {low }} \mathrm{CD} 25^{\text {low }}$ population, are also prevalent in autoimmunity and likely to be involved in FOXP3 turnover as well (40). We also note the modest sample size and the lack of data from diseased tissues; hence, these data warrant further investigation in a validation study with a large cohort of patients, by using multiparametric labeling, longitudinal samples, and functional assays to establish the frequency and the cell type specificity of target expression. Similarly, further work will be needed to assess the roles of additional miRs, including miR-9-5p, which did not reach statistical significance in our analysis.

In conclusion, our preliminary results implicate a potentially significant role of T-bet/miR-29a-3p axis and their cellular sources in HT presentation. The results broaden our understanding of transcriptional alterations in HT, and link T-bet/miR-29a-3p pathways with the heterogeneity of clinical and laboratory manifestations among HT patients. Statistical power remains limited in small datasets; thus, more work is needed, preferably in well-powered datasets, to consolidate observed differences and clarify specific roles for the miRNA in the actual mechanisms of HT pathogenesis.

\section{REFERENCES}

1. Pearce EN, Farwell AP, Braverman LE. Thyroiditis. N Engl JMed (2003) 348(26):2646-55. doi:10.1056/NEJMra021194

2. Shi Y, Wang H, Su Z, Chen J, Xue Y, Wang S, et al. Differentiation imbalance of Th1/Th17 in peripheral blood mononuclear cells might contribute to pathogenesis of Hashimoto's thyroiditis. Scand J Immunol (2010) 72(3):250-5. doi:10.1111/j.1365-3083.2010.02425.x

3. Tokić S, Štefanić M, Glavaš-Obrovac L, Jaman S, Novosadová E, Petrkova J, et al. The expression of T cell FOXP3 and T-bet is upregulated in severe but not euthyroid Hashimoto's thyroiditis. Mediators Inflamm (2016) 2016:3687420. doi:10.1155/2016/368742

4. Figueroa-Vega N, Alfonso-Pérez M, Benedicto I, Sánchez-Madrid F, GonzálezAmaro R, Marazuela M. Increased circulating pro-inflammatory cytokines and Th17 lymphocytes in Hashimoto's thyroiditis. J Clin Endocrinol Metab (2010) 95(2):953-62. doi:10.1210/jc.2009-1719

5. Liu Y, Tang X, Tian J, Zhu C, Peng H, Rui K, et al. Th17/Treg cells imbalance and GITRL profile in patients with Hashimoto's thyroiditis. Int J Mol Sci (2014) 15(12):21674-86. doi:10.3390/ijms151221674

6. Li C, Yuan J, Zhu YF, Yang XJ, Wang Q, Xu J, et al. Imbalance of Th17/Treg in different subtypes of autoimmune thyroid diseases. Cell Physiol Biochem (2016) 40(1-2):245-52. doi:10.1159/000452541

7. Marazuela M, García-López MA, Figueroa-Vega N, de la Fuente H, AlvaradoSánchez B, Monsiváis-Urenda A, et al. Regulatory T cells in human autoimmune thyroid disease. JClin Endocrinol Metab (2006) 91(9):3639-46. doi:10.1210/jc.2005-2337

8. Xue H, Yu X, Ma L, Song S, Li Y, Zhang L, et al. The possible role of $\mathrm{CD} 4^{+} \mathrm{CD} 25^{\text {high }}{ }^{\mathrm{Foxp}} 3^{+} / \mathrm{CD} 4^{+} \mathrm{IL}-17 \mathrm{~A}^{+}$cell imbalance in the autoimmunity of patients with Hashimoto thyroiditis. Endocrine (2015) 50(3):665-73. doi:10.1007/s12020-015-0569-y

9. Kishore A, Borucka J, Petrkova J, Petrek M. Novel insights into miRNA in lung and heart inflammatory diseases. Med Inflamm (2014) 259131:27. doi:10.1155/2014/259131

10. Steiner DF, Thomas MF, Hu JK, Yang Z, Babiarz JE, Allen CD, et al. MicroRNA-29 regulates T-box transcription factors and interferon- $\gamma$

\section{ETHICS STATEMENT}

This study was carried out in accordance with the recommendations of the institutional ethical committee of the Osijek University Hospital, with written informed consent from all subjects, and in accordance with the Declaration of Helsinki. The protocol was approved by the institutional ethical committee of the Osijek University Hospital.

\section{AUTHOR CONTRIBUTIONS}

Conceived and designed the experiments: MP, ST, and MŠ. Performed the experiments: ST, MŠ, LG-O, AK, and ZN. Analyzed the data: ST and MŠ. Contributed reagents/materials/ analysis tools: MP, MŠ, and LG-O. Contributed to the writing of the manuscript: ST, MŠ, and MP. All the authors reviewed and approved the final manuscript.

\section{FUNDING}

This work was supported by the Croatian Ministry of Science, Education and Sports (Grant No. 219-2190372-2068), Josip Juraj Strossmayer University of Osijek (VIF2016MEFOS-37), and Palacky University Olomouc (IGA_LF_2017_014).

production in helper T cells. Immunity (2011) 35(2):169-81. doi:10.1016/j. immuni.2011.07.009

11. Fayyad-Kazan H, Rouas R, Fayyad-Kazan M, Badran R, El Zein N, Lewalle P, et al. MicroRNA profile of circulating CD4-positive regulatory $\mathrm{T}$ cells in human adults and impact of differentially expressed microRNAs on expression of two genes essential to their function. J Biol Chem (2012) 287(22):18584. doi:10.1074/jbc.M111.337154

12. Jebbawi F, Fayyad-Kazan H, Merimi M, Lewalle P, Verougstraete J-C, Leo $\mathrm{O}$, et al. A microRNA profile of human $\mathrm{CD}^{+}$regulatory $\mathrm{T}$ cells and characterization of the effects of microRNAs on Treg cell-associated genes. J Transl Med (2014) 12:218. doi:10.1186/s12967-014-0218-x

13. Wang $H$, Flach $H$, Onizawa M, Wei L, McManus MT, Weiss A. Negative regulation of Hifla expression and TH17 differentiation by hypoxia regulated miR-210. Nat Immunol (2014) 15(4):393-401. doi:10.1038/ni.2846

14. Dang EV, Barbi J, Yang HY, Jinasena D, Yu H, Zheng Y, et al. Control of $\mathrm{T}(\mathrm{H}) 17 / \mathrm{T}(\mathrm{reg})$ balance by hypoxia-inducible factor 1. Cell (2011) 146(5):772-84. doi:10.1016/j.cell.2011.07.033

15. Štefanić M, Papić S, Suver M, Glavaš-Obrovac L, Karner I. Association of vitamin D receptor gene 3' variants with Hashimoto's thyroiditis in the Croatian population. Int J Immunogenet (2008) 35(2):125-31. doi:10.1111/j.1744313X.2008.00748.x

16. Tokic S, Štefanić M, Karner I, Glavaš-Obrovac L. Altered expression of CTLA4, CD28, VDR, and CD45 mRNA in T cells of patients with Hashimoto's thyroiditis - a pilot study. Endokrynol Pol (2017) 68(3):274-82. doi:10.5603/ EP.2017.0010

17. Chomczynski P, Sacchi N. Single-step method of RNA isolation by acid guanidinium thiocyanate-phenol-chloroform extraction. Anal Biochem (1987) 162:156-9. doi:10.1006/abio.1987.9999

18. Pfaffl MW. A new mathematical model for relative quantification in realtime RT-PCR. Nucleic Acids Res (2001) 29(9):2002-7. doi:10.1093/nar/ 29.9.e45

19. Novosadova E, Chabronova A, Kolek V, Petrek M, Navratilova Z. The serum expression of selected miRNAs in pulmonary sarcoidosis with/without Löfgren's syndrome. Med Inflamm (2016) 2016:1246129. doi:10.1155/2016/ 1246129 
20. Jakobs TC, Mentrup B, Schmutzler C, Dreher I, Köhrle J. Proinflammatory cytokines inhibit the expression and function of human type I 5'-deiodinase in HepG2 hepatocarcinoma cells. Eur J Endocrinol (2002) 146(4):559-66. doi:10.1530/eje.0.1460559

21. Xu G, Tu W, Qin S. The relationship between deiodinase activity and inflammatory responses under the stimulation of uremic toxins. J Transl Med (2014) 12:239. doi:10.1186/s12967-014-0239-5

22. Gérard AC, Boucquey M, van den Hove MF, Colin IM. Expression of TPO and ThOXs in human thyrocytes is downregulated by IL-1-alpha/IFN-gamma, an effect partially mediated by nitric oxide. Am J Physiol Endocrinol Metab (2006) 291(2):E242-53. doi:10.1152/ajpendo.00439.2005

23. Marique L, Van Regemorter V, Gérard AC, Craps J, Senou M, Marbaix E, et al. The expression of dual oxidase, thyroid peroxidase, and caveolin-1 differs according to the type of immune response (TH1/TH2) involved in thyroid autoimmune disorders. JClin Endocrinol Metab (2014) 99(5):1722-32. doi:10.1210/jc.2013-3469

24. Mazziotti G, Sorvillo F, Naclerio C, Farzati A, Cioffi M, Perna R, et al. Type-1 response in peripheral CD4 and CD8 T cells from patients with Hashimoto's thyroiditis. Eur J Endocrinol (2003) 148:383-8. doi:10.1530/eje.0.1480383

25. Karanikas G, Schuetz M, Wahl K, Paul M, Kontur S, Pietschmann P, et al. Relation of anti-TPO autoantibody titre and T-lymphocyte cytokine production patterns in Hashimoto's thyroiditis. Clin Endocrinol (Oxf) (2005) 63(2):191-6. doi:10.1111/j.1365-2265.2005.02324.x

26. Bossowski A, Harasymczuk J, Moniuszko A, Bossowska A, Hilczer M, Ratomski K. Cytometric evaluation of intracellular IFN-g and IL-4 levels in thyroid follicular cells from patients with autoimmune thyroid diseases. Thyroid Res (2011) 4:13. doi:10.1186/1756-6614-4-13

27. Liu Y, You R, Yu N, Gong Y, Qu C, Zhang Y, et al. Increased proportions of Tc17 cells and NK cells may be risk factors for disease progression in Hashimoto's thyroiditis. Int Immunopharmacol (2016) 40:332-8. doi:10.1016/j.intimp.2016.09.016

28. Brain O, Owens BM, Pichulik T, Allan P, Khatamzas E, Leslie A, et al. The intracellular sensor NOD2 induces microRNA-29 expression in human dendritic cells to limit IL-23 release. Immunity (2013) 39(3):521-36. doi:10.1016/j. immuni.2013.08.035

29. Mikoś H, Mikoś M, Obara-Moszyńska M, Niedziela M. The role of the immune system and cytokines involved in the pathogenesis of autoimmune thyroid disease (AITD). Endokrynol Pol (2014) 65(2):150-5. doi:10.5603/EP.2014.0021

30. Kiyici S, Gul OO, Baskan EB, Hacioglu S, Budak F, Erturk E, et al. Effect of levothyroxine treatment on clinical symptoms and serum cytokine levels in euthyroid patients with chronic idiopathic urticaria and thyroid autoimmunity. Clin Exp Dermatol (2010) 35:603-7. doi:10.1111/j.1365-2230. 2009.03642.x

31. Xin A, Masson F, Liao Y, Preston S, Guan T, Gloury R, et al. A molecular threshold for effector CD8+ T cell differentiation controlled by transcription factors Blimp-1 and T-bet. Nat Immunol (2016) 17:422-32. doi:10.1038/ni.3410
32. Kallies A, Xin A, Belz GT, Nutt SL. Blimp-1 transcription factor is required for the differentiation of effector CD8+ T cells and memory responses. Immunity (2009) 31:283-95. doi:10.1016/j.immuni.2009.06.021

33. Luo J, Niu X, Zhang M, Zhang K, Chen M, Deng S. Inhibition of B lymphocyte-induced maturation protein-1 reduces the production of autoantibody and alleviates symptoms of systemic lupus erythematosus. Autoimmunity (2015) 48(2):80-6. doi:10.3109/08916934.2014.976627

34. Boulet S, Daudelin J-F, Labrecque N. IL-2 induction of Blimp-1 is a key in vivo signal for CD8+ short-lived effector T cell differentiation. J Immunol (2014) 193(4):1847-54. doi:10.4049/jimmunol.1302365

35. Lazarevic V, Chen X, Shim J-H, Hwang ES, Jang E, Bolm AN, et al. T-bet represses TH17 differentiation by preventing Runx1-mediated activation of the gene encoding ROR $\gamma$ t. Nat Immunol (2016) 12:96-104. doi:10.1038/ ni.1969

36. Wang Y, Godec J, Ben-Aissa K, Cui K, Zhao K, Pucsek AB, et al. The transcription factors T-bet and Runx are required for the ontogeny of pathogenic interferon- $\boldsymbol{\gamma}$-producing T helper 17 cells. Immunity (2014) 40(3):355-66. doi:10.1016/j.immuni.2014.01.002

37. Teteloshvili N, Smigielska-Czepiel K, Kroesen BJ, Brouwer E, Kluiver J, Boots AM, et al. T-cell activation induces dynamic changes in miRNA expression patterns in CD4 and CD8 T-cell subsets. Microrna (2015) 4(2):117-22. doi: $10.2174 / 2211536604666150819194636$

38. Haoquan W, Neilson JR, Kumar P, Manocha M, Shankar P, Sharp PA, et al. miRNA profiling of naïve, effector and memory CD8 T cells. PLoS One (2007) 2(10):e1020. doi:10.1371/journal.pone.0001020

39. Lai X, Wolkenhauer O, Vera J. Understanding microRNA-mediated gene regulatory networks through mathematical modelling. Nucleic Acids Res (2016) 44(13):6019-35. doi:10.1093/nar/gkw550

40. Ferreira RC, Simons HZ, Thompson WS, Rainbow DB, Yang X, Cutler AJ, et al. Cells with Treg-specific FOXP3 demethylation but low CD25 are prevalent 2 in autoimmunity. J Autoimmun (2017) 84:75-86. doi:10.1101/ 134692

Conflict of Interest Statement: The authors declare that the research was conducted in the absence of any commercial or financial relationship that could be construed as a potential conflict of interest.

Copyright (C) 2018 Tokić, Štefanić, Glavaš-Obrovac, Kishore, Navratilova and Petrek This is an open-access article distributed under the terms of the Creative Commons Attribution License (CC BY). The use, distribution or reproduction in other forums is permitted, provided the original author(s) and the copyright owner are credited and that the original publication in this journal is cited, in accordance with accepted academic practice. No use, distribution or reproduction is permitted which does not comply with these terms. 